Florencia Lloret y una mirada al fondo documental de Adelina Dematti de Alaye

María Emilia Nieto

http://perio.unlp.edu.ar/ojs/index.php/question/article/view/4715

Cita sugerida: Nieto, M. (2018). Florencia Lloret y una mirada al fondo documental de Adelina Dematti de Alaye.

Question, 1(59), e087. doi:https://doi.org/10.24215/16696581e087

\title{
Florencia Lloret y una mirada al fondo documental de Adelina Dematti de Alaye
}

\author{
Florencia Lloret and a look at the documentary collection of Adelina \\ Dematti de Alaye
}

María Emilia Nieto mariaemilianieto@gmail.com

http://orcid.org/0000-0002-8078-3441

Instituto de Investigaciones en Humanidades y Ciencias Sociales; Universidad Nacional de La Plata/ Consejo Nacional de Investigaciones Científicas y Técnicas (Argentina)

\section{Resumen}

Entrevista realizada a Florencia Lloret, museóloga e integrante del sector Historia Reciente del Archivo histórico de la provincia de Buenos Aires, ubicado en la ciudad de La Plata. La finalidad del archivo consiste en reunir los fondos documentales existentes en las oficinas públicas de la provincia, con el objetivo de difundir el patrimonio cultural y acercar estos documentos a la comunidad de investigadores y al conjunto de la ciudadanía. Nuestro interés consistió en abordar centralmente el fondo documental de Adelina Dematti de Alaye, integrante de Madres de Plaza de Mayo, La Plata, que se encuentra en la sección dedicada a la Historia Reciente.

Palabras clave: historia reciente; memoria; Madres de Plaza de Mayo. 


\begin{abstract}
Interview with Florencia Lloret, museologist and member of the Recent History Sector of the Historical Archive of Provincia de Buenos Aires, located in La Plata. The purpose of the archive is to gather the existing documentary funds in the public offices of the province, with the aim of disseminating the cultural heritage and bringing these documents to the community of researchers and to the citizenry as a whole. Our interest was to address centrally the documentary background of Adelina Dematti de Alaye, member of Madres de Plaza de MayoLa Plata, which is in the Section dedicated to recent history.
\end{abstract}

Keywords: recent history; memoirs; Madres de Plaza de Mayo.

Florencia Lloret es museóloga, integrante del sector Historia Reciente del Archivo Histórico de la provincia de Buenos Aires, ubicado en la ciudad de La Plata, cuya finalidad consiste en reunir los fondos documentales existentes en las oficinas públicas de la provincia, con el objetivo de difundir el patrimonio cultural y acercar estos documentos a la comunidad de investigadores y al conjunto de la ciudadanía. El archivo cuenta con una sección dedicada a la Historia Reciente, en la que se encuentra el fondo documental de Adelina Dematti de Alaye, integrante de Madres de Plaza de Mayo-La Plata. Declarado Patrimonio de la UNESCO en 2007, el archivo fue donado por Adelina con el interés de garantizar su acceso público.

\title{
-¿Qué es el Archivo Histórico Provincial, y qué lugar ocupa el archivo de Adelina Dematti de Alaye?
}

- Los fondos del Archivo Histórico Provincial abarcan desde fines del siglo XVII hasta el siglo $X X$, aunque el período de mayor densidad documental corresponde al siglo XIX y es documentación emanada de organismos públicos, de los ministerios básicamente, de lo que eran antes otras instituciones públicas. Se crea en 1925, a cargo del historiador Ricardo Levene con el objetivo de reunir los fondos de la administración pública provincial. Durante mucho tiempo el Archivo tuvo una línea de investigación histórica que de alguna manera abrió el juego cuando se empezaron a hacer los Congresos de Historia de los Pueblos de la provincia de Buenos Aires; ahí hay una apertura a un reconocimiento a historiadores locales, a 
incorporar una historia más cotidiana, otro tipo de historia que no es sólo la del documento histórico que conserva el Archivo. El Primer Congreso de Historia de los Pueblos fue iniciativa de Levene. Después se discontinúan durante unos años y se retoman luego ya ininterrumpidamente cada dos años. En 2000 aparecen dos cuestiones en el Archivo que son claves: una es la incorporación de las exposiciones al Archivo Histórico, las exposiciones temporarias como una forma de difusión de la historia, de llegar al público que no es asiduo, que no es investigador. Las exposiciones en ese punto son una herramienta de difusión y muy interesante para trabajar. Hay un equipo armado de museología dentro del Archivo Histórico, que nació en esa instancia, cuando el Archivo cumple 75 años. Allí se hace una exposición sobre el Archivo, sobre su historia, y queda conformado un equipo de trabajo en función de las exposiciones itinerantes que recorrían el interior de la provincia de Buenos Aires con documentos originales. Lo que siempre tratamos de preservar es llevar al interior de la Provincia lo mismo que se podía ver acá. Tratar siempre de llevar el documento original, si bien era un esfuerzo y una responsabilidad muy grande para nosotros, permitía que el que estuviera en el interior de la Provincia pudiera ver lo mismo que el que estaba en La Plata.

Entonces, por un lado las exposiciones y por otro, la incorporación de la sección Historia Reciente dentro del Archivo Histórico. En esta sección trabajamos con la historia oral para generar documentos a partir del relato de memorias individuales y la construcción de memorias colectivas, como posibilidad de análisis. Se hicieron muchos talleres de historia oral, por ejemplo sobre el ferrocarril, sobre docencia. Después fueron temas más concretos, y entrevistas individuales como las de ex combatientes de Malvinas. Con el CECIM La Plata hay un convenio hecho y un libro publicado con sus entrevistas. Con Madres de Plaza de Mayo-La Plata se firmó un convenio para empezar a entrevistarlas. La relación la hicimos con Adelina primero, cuando ella trabajaba en la Secretaría de Derechos Humanos de la Municipalidad.

Como toda entrevista de historia oral, son extensas; lo que tratábamos de buscar era siempre la historia previa a que se convirtieran en Madres; el momento de ser Madres de Plaza de Mayo, su mirada actual sobre eso también y sus búsquedas actuales.

En todo ese recorrido Adelina no quería que la entrevistáramos y en 2007 el Archivo de Adelina es declarado Memoria del Mundo por la UNESCO. Y en ese momento decide que su archivo tenía que ser donado a alguna institución y decidió donarlo al Archivo de la Provincia, en 2008. 


\section{— ¿Cómo fue el proceso de creación del archivo? ¿O ya estaba creado?}

—Antes de 2008 muchas veces aparecía Adelina y nombraban "Archivo personal Adelina Dematti de Alaye"; ella tenía su archivo personal en el garaje de su casa. Tenemos algunas fotos de su archivo en ese momento, tenía cajas, cajas y cajas, y sólo ella sabía lo que tenía ahí. Podías ir, llamarla por teléfono, decirle que estabas investigando sobre tal cosa y ella te abría las puertas, te daba documentación, así es como también ha perdido documentación, cosas que sabía que ella había prestado y te decía "fulanito nunca me lo trajo". Ella generó ese archivo a partir de sus búsquedas, o de la búsqueda de Carlos, puntualmente, y todo su trabajo posterior. Ese archivo existía. Lo que decide, a partir de ser declarado Memoria del Mundo, es donarlo al Archivo Histórico con una clara fundamentación, que era que todo lo que ella había ido guardando tenía que tener sentido y estar al alcance de cualquier persona que quisiera visitarlo. Consideraba que otros archivos $u$ otras instituciones privadas quizás no iban a permitir esa posibilidad. Era su mirada sobre el tema, y pensó que el Archivo Histórico de la Provincia era el mejor lugar para donarlo. Eso ingresó en una sección que se llama Historia Reciente, conformada por otros fondos, y pasa a ocupar una parte absolutamente importante, y muchas veces nosotros mismos lo seguimos llamando Archivo Personal Adelina Alaye, es como hasta un reconocimiento que uno hace. Pero la sección Historia Reciente conforma una cantidad de fondos más grandes. El fondo Mercante que fue el gobernador de la provincia de Buenos Aires en época del primer gobierno peronista (1946-1952), tiene un montón de colecciones porque se han hecho entrevistas de historia oral, se han donado objetos, no sólo de Mercante, sino de ministros que pertenecieron a esa gobernación; también documentación de un periodista de la provincia, Senén González; del CECIM están las entrevistas de historia oral que constituyen para nosotros un documento. Asociado a eso hay fotografías, digitalización de fotos pertenecientes a ex combatientes, para nosotros eso constituye parte de esa colección, hay diarios del momento de la guerra también. Está el Fondo fotográfico del diario El Argentino de La Plata; documentación y libros pertenecientes a Emilio Mignone; documentación relacionada con la República de los Niños. El Archivo de Adelina se incluye dentro de una sección que es Historia Reciente que es más amplia. 


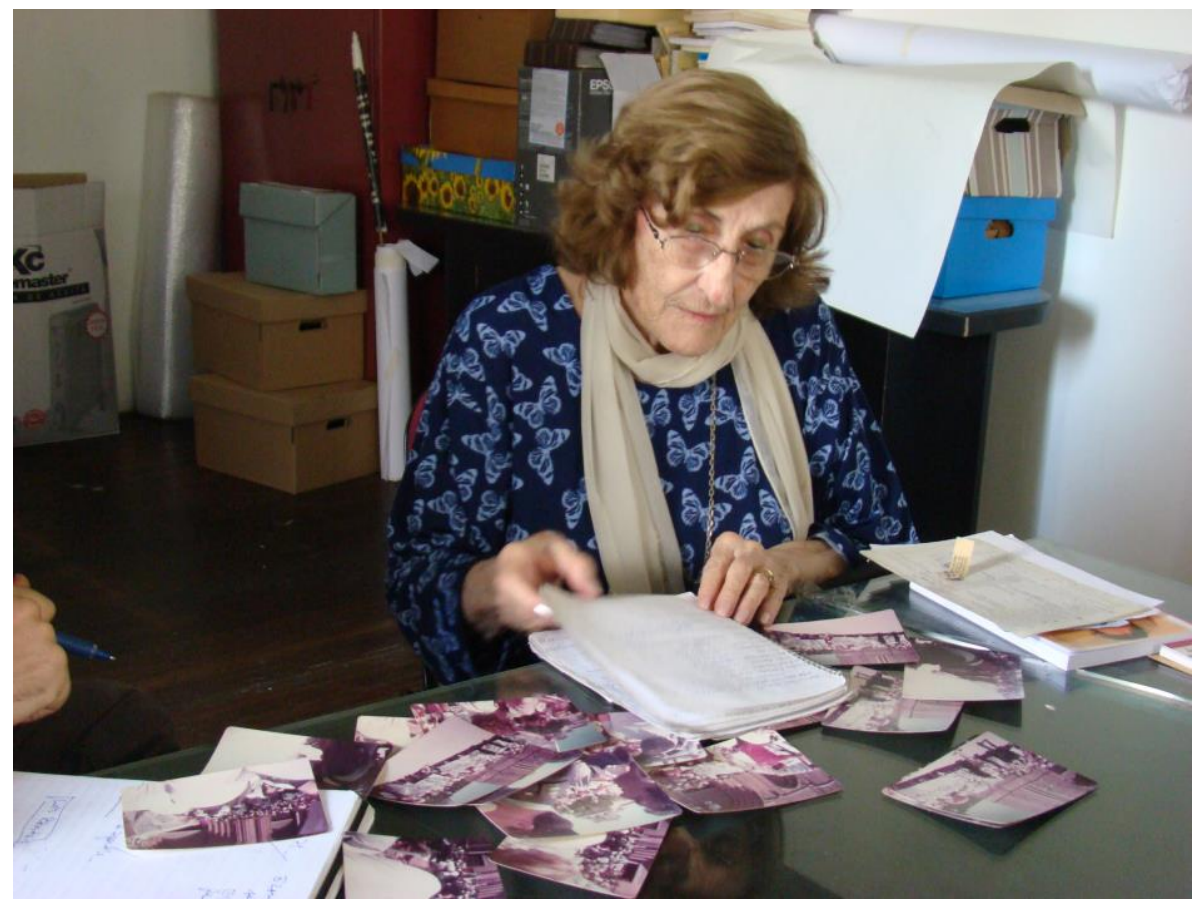

Foto 1. Adelina trabajando con sus fotografías

\section{— ¿Cómo fue el proceso de organización de ese material?}

-El archivo de Adelina tenía una primera organización que era sin duda la que ella le había dado a lo largo del tiempo. Cuando ingresa al Archivo Histórico había sufrido parte de una digitalización y parte de un ordenamiento, por Memoria Abierta. Memoria Abierta tenía un programa de archivos sobre Derechos Humanos, sobre delitos de lesa humanidad, en toda Latinoamérica, entonces un equipo a cargo de un especialista brasilero estuvo trabajando con el archivo de Adelina, en la producción de una herramienta descriptiva. Esa herramienta básicamente da cuenta de un ordenamiento posible, en series, subseries, en temas, y un poco reordenaron esa documentación que Adelina tenía. Ingresa de esa manera al Archivo Histórico, con un pre ordenamiento. Cuando nosotros empezamos a trabajar con la documentación, encontramos que había cosas que desde la producción de la herramienta descriptiva, desde el escrito tenían un sentido y cuando ibas a los documentos no llegaba a ese mismo sentido, como que el ordenamiento no guardaba el mismo sentido que el escrito, que la herramienta. Entonces lo que tratamos fue, con el acuerdo de Memoria Abierta, que se guardara ese correlato entre la herramienta descriptiva y lo que nosotros encontrábamos. Y fuimos 
profundizando esa herramienta descriptiva, en algunos casos tratamos que fuera lo mínimo, en otros tuvimos que cambiar algunas divisiones, porque considerábamos que era más adecuado, y en otros casos permaneció esa división, pero lo que hicimos fue empezar a darle un ordenamiento y un sentido a los documentos. Los cambios que se produjeron casi todas las veces tuvieron que ver con preguntas que nosotros le hacíamos a Adelina: ¿Qué es este papel? ¿Por qué está este documento acá? Entonces cuando ella te empezaba a contar la historia, a darle sentido, encontrábamos que no era sólo el documento, que por ejemplo había nacido de un habeas corpus, era un documento que había nacido en esa instancia pero que en realidad tenía relación con un caso que Adelina venía investigando, de un profesor por ejemplo, como es el caso de Eguía, que a ella le aparece en la historia de su vida muchas veces desde la docencia y luego desde la búsqueda de Carlos. No porque estuviera relacionado con Carlos directamente, sino con desapariciones o con datos que él, como profesor o preceptor de la escuela, daba a los servicios de inteligencia. Hay muchos casos donde el documento tiene un membrete y vos decís "lo tengo que asociar con tal serie o con tal proceso judicial, o con tal ministerio" porque deviene de ese lugar, y cuando Adelina te contaba porqué existía ese documento le empezabas a dar otro sentido y lo tenías que ubicar en otro lado, necesariamente. Eso fue lo que muchas veces nos pasó, y de hecho todavía seguimos trabajando en eso. Es un trabajo muy extenso que estamos haciendo para tratar de dejarlo ordenado. Es complejo porque a medida que te vas metiendo en el archivo van apareciendo otras cosas, es inevitable.

\section{—Pero fueron construyendo esa organización con Adelina...}

-Sí, todo el proceso se trabajó mucho con Adelina. Por un lado se la empezó a entrevistar semanalmente. Tenemos un trabajo publicado que se llama "Los martes Adelina" y que es justamente sobre el trabajo de historia oral que estábamos haciendo con ella. Era juntarnos todos los martes y proponerle temáticas o puntos sobre los cuales pudiera ir hablando, pero básicamente con la intención de que pudiera ir dando cuenta de su trayectoria de vida y en esos relatos aparecían los documentos permanentemente, o mencionaba "debe estar en mi archivo", o nosotros a medida que íbamos avanzando le íbamos preguntando constantemente. Y nos quedó muchísimo sin preguntarle, obviamente, cantidad, horrores. Pero bueno, era parte de lo que uno sabía, era inevitable. Una línea fue entonces en función de eso. Y otra línea, que en esa también hay muchas entrevistas, es sobre lo que más avanzado está el ordenamiento 
de su archivo: las fotografías. Ya cuando no la entrevistamos más o abandonamos un poco las entrevistas de historia oral directamente a partir de su vida, lo que empezamos a hacer fue a trabajar sobre sus fotografías. Y ahí sí, las fotografías estaban digitalizadas en un 80\% por Memoria Abierta. Digo en un $80 \%$ porque una vez que el archivo ingresa acá, empiezan a aparecer cantidades de fotos que Adelina tenía guardadas en su casa, o fotos que después fue sacando con sus cámaras digitales y empezaron a aparecer discos con fotos. De lo que ingresó, había un porcentaje digitalizado que nos vino fantástico, que eran las fotos que tenían más que ver con los setenta, o a partir de 1977 que son las primeras búsquedas de Carlos o cuando desaparece Carlos, y cuando ella se incorpora a Madres. Pero hay toda una etapa previa que tiene que ver con su vida personal, y toda una etapa posterior.

\section{— ¿La fotografía formaba parte de su vida, previamente?}

- Hay fotografías de Adelina que dan cuenta de que agarraba la cámara y le gustaba sacar fotos. Yo siempre digo que aparecieron unas fotos color, era como un desfile, que podía parecer un día de la tradición, un acto en algún lado, y cuando le preguntamos qué era se empezó a matar de risa, ni se acordaba exactamente pero dice "debía ser en Chivilcoy, en algún acto". Fotos a sus pañuelos en la casa - a ella le encantaban los pañuelos- a las flores. Indudablemente tenía un interés en sacar fotos. Después, a partir de que empieza a integrar Madres de Plaza de Mayo, lo que ella dice es que llevaba su cámara escondida y sí, ya con fines de poder registrar esas situaciones, en una cuestión muy arriesgada que era tratar de sacar una cámara. Entonces hay un montón de fotos que, desde el punto de vista fotográfico, no tienen calidad, no son buenas fotos. Lo que es interesante es en función de lo que relata esa foto, aunque esté fuera de foco, aunque esté movida. Hay tres fotos que tengo muy presentes que fue cuando vino la Comisión Interamericana de Derechos Humanos (CIDH) de la OEA a la Argentina, en septiembre de 1979, y atendió a familiares de víctimas o desaparecidos en un espacio en Avenida de Mayo. Hay tres fotos que parece que puede ser en una calle de Buenos Aires, bastante fuera de foco y se ve una cantidad de gente, en una fila, pero está tomada desde la vereda de enfrente. De ninguna manera dirías que es una buena foto, y cuando le preguntamos a ella, dijo que eran las tres fotos de la gente haciendo la cola para entrar a entrevistarse con la gente de la OEA. ¡Como testimonio es importantísima! Hay mucho registro fotográfico de Adelina que cae en ese lugar, estéticamente no vas a encontrar las mejores fotos -algunas sí- pero en líneas generales en ese periodo de ella en Madres, lo que 
hay es una necesidad de registro de lo que estaba sucediendo, de dónde estaban, de cubrirse de alguna manera también ellas. $Y$ cuando ya regresa la democracia, lo que uno de alguna manera también observa es que Adelina sigue con esa necesidad de continuar el registro, de los lugares donde está. Entonces tenía una política: cuando iba a un acto, sacaba las fotos que creía que servían, que le interesaban o lo que fuera, y después siempre pedía que le sacaran una foto a ella, daba la cámara y pedía que le sacaran una foto, como dejar el testimonio de que había estado en ese lugar. También hay toda una serie de fotos que saca a los camarógrafos que están filmando o sacando fotos en determinados actos.

Indudablemente había un interés por la fotografía, sin duda. En su Archivo también hay muchas fotos que no ha sacado ella. En proporción, digamos que es menor que el porcentaje de fotos que ha sacado ella, que tienen que ver sobre todo con las fotos más familiares, de su mamá, de sus hermanas, de sus hermanos, de su marido, pero que sin duda forman parte de su archivo personal y en este caso de su archivo fotográfico.

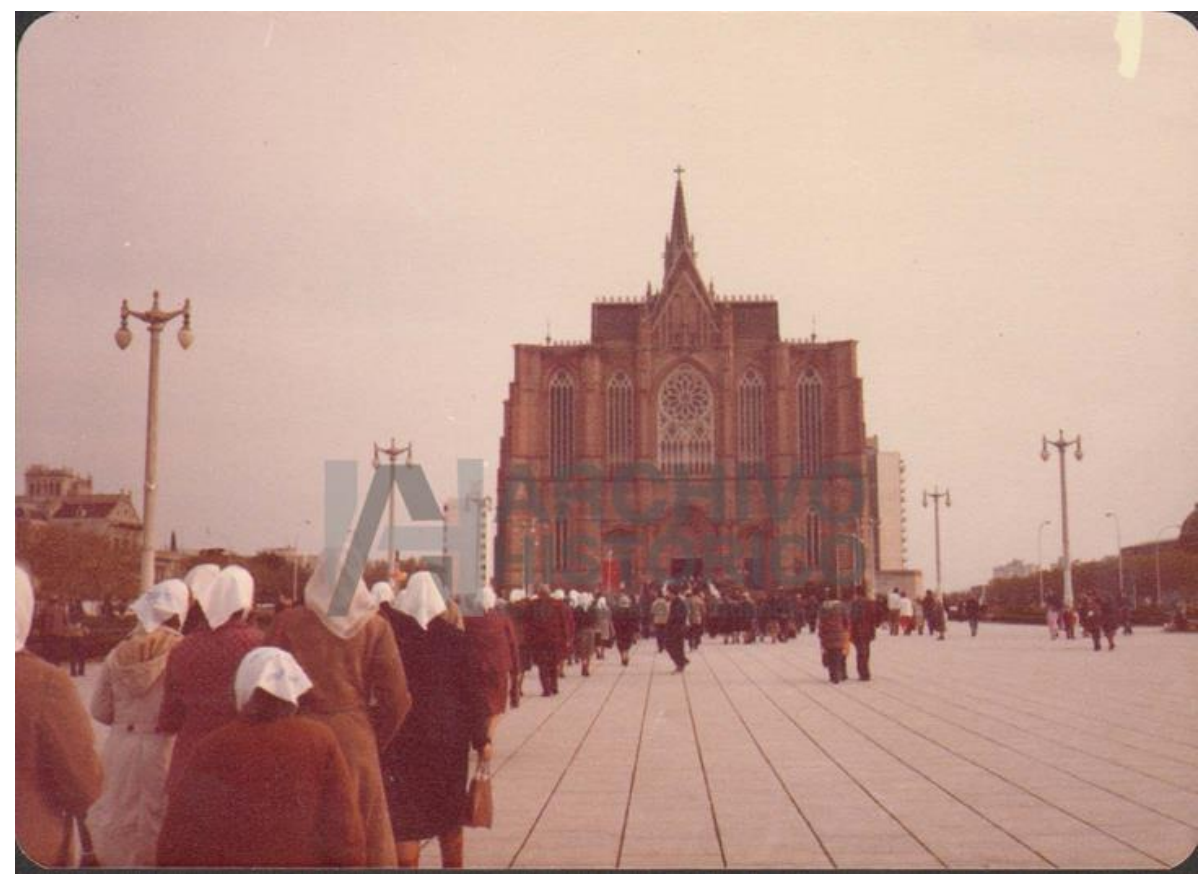

Foto 2. Procesión Corpus Christi, Catedral de La Plata. "Procesión de corpus, La Plata, 16/6/80. Las que llevaban pañuelo en el brazo eran por otras flias." (Fondo documental de Adelina Dematti de Alaye, integrante de Madres de Plaza de Mayo-La Plata). 16 de junio de 1980. 


\section{-Si bien ya fuiste mencionando algunos ¿qué criterios se utilizaron para organizar el archivo?}

- Los criterios de organización que se utilizaron de alguna manera devienen de lo que estaba hecho por Memoria Abierta. Nosotros tratamos de tomar lo que es Adelina Dematti de Alaye como una persona que tiene una historia previa a ser Madre de Plaza de Mayo. Entonces se encuentra una serie con su documentación personal, tanto de ella como de su familia. Hay actas de defunción de parientes, actas de nacimiento de sus hijos, certificados de bautismo, etcétera. En esa serie también se encuentra todo lo que tiene que ver con correspondencia, las cartas dirigidas a Adelina. $Y$ ahí hay un gran sector que no está abierto al público, que tiene que ver con la correspondencia de su hija, de su nuera y de su nieta desde el exilio, que es una de las cuestiones que el Archivo Histórico de la Provincia tiene: una restricción sobre eso, porque considera que es una documentación muy sensible con todas esas personas que viven aún. Por eso es parte de la documentación que no está abierta al público, al igual que las fotografías que tienen que ver con su vida personal, con sus nietos, con la vida más cercana, más íntima, más privada. Pero sí están incorporadas a estas divisiones, a estas organizaciones. Entonces, una primer etapa que tiene que ver con Adelina como personaje, y ahí está la parte de docencia, todo su desarrollo como docente desde que se recibe; ella fue docente en el nivel inicial, en primaria, inspectora, preceptora, directora de colegios, pasó por muchas instancias y se encargó de guardar toda esa documentación.

\section{-En esos documentos hay una reflexión sobre su práctica docente y su formación}

-Sí, hay mucha producción propia. Adelina nunca dejó de producir material sobre aquello que estaba haciendo. Tanto en la etapa de docente, con sus dos hijos y siendo directora de un colegio en Brandsen, a la etapa en la que ya está en la búsqueda de Carlos y posteriormente, siempre hay producción de Adelina. Siempre hay un momento de reflexión y de sentarse a escribir sobre lo que está haciendo. En el caso de la docencia hay muchísimo, desde discursos hasta material pedagógico escrito por ella. Hay incluso un proyecto que presentó sobre escuelas piloto. Siendo directora genera actividades alrededor de un centro cultural, y de gente que viene y que va de La Plata y que ella lleva a Brandsen, hay siempre un interés más allá de su profesión. 
Todo lo que nosotros llamamos "manuscritos de Adelina" son cúmulos y cúmulos de papelitos. En una libretita donde había anotado de un lado, la lista de las compras; del otro una dirección y un dato y una reflexión que ella hizo. Ella decía que nunca había podido leer un discurso largo sin saltearse ninguna parte; en sus discursos, siempre encontrás anotaciones, apuntes. Dentro de la serie personal, correspondencia y docencia son dos ejes importantes. Hay otra serie que es "Carlos Alaye", donde se agrupa toda la documentación que da cuenta de Carlos de niño, antes de su desaparición, en el momento que desaparece y las búsquedas que hace Adelina, judiciales y no judiciales, en el país y en el exterior. Luego hay otra serie que es lo que nosotros llamamos "militancia orgánica", y tiene que ver con todas las acciones y todos los grupos de militancia de Adelina.

Adelina estuvo en Madres de Plaza de Mayo-La Plata, pero también estuvo en la Comisión Provincial por la Memoria cuando se funda; fue miembro de la APDH, y de otras comisiones y agrupaciones de las que participó activamente. En toda esa serie de militancia está incorporado lo que tiene que ver con las búsquedas, ya no de Carlos en particular, sino de otros desaparecidos. Se abre un mundo mientras ella sigue buscando a Carlos, se involucra en búsquedas más colectivas. En esa serie están los documentos que dan cuenta de la investigación que ella hace en el cementerio de La Plata que produce, tiempo después, un libro. Están los listados de desaparecidos por facultades también. Todo eso está en militancia organizada.

$Y$ después hay otra sección que tiene que ver con militancia $u$ otro tipo de militancia que no es tan organizada, todo el trabajo posterior que ha hecho Adelina en el tiempo, desde por ejemplo la Secretaría de Derechos Humanos de La Plata donde trabajaba ad honorem, y desde otros estamentos también, donde lo que hacía era trabajar con las cárceles, trabajar con situaciones problemáticas en escuelas, derechos humanos en general. Hay cuestiones que tienen que ver con la militancia en la vida de Adelina, posterior a la búsqueda de su hijo, aunque siempre lo siguió buscando.

La particularidad del archivo de Adelina, es que tiene documentos escritos, gráficos, muchas revistas. Tiene libros, donó gran parte de su biblioteca. Hay novelas que leía de joven, libros que tienen que ver con política y con derechos humanos, más viejos y más actuales. Sin duda su biblioteca no está completa, eso fue lo que ella eligió y quiso donar. Su archivo también está compuesto por los documentos sonoros que son todas las entrevistas que nosotros generamos (con distintos niveles de accesibilidad); y también hay mucho en soporte digital: videos, $\mathrm{Cd}$, DVD, VHS. Y hay objetos que ella donó, por ejemplo, los zapatitos de sus hijos de cuando recién nacieron, juguetes que eran de Carlos, soldaditos, autitos. Hay toda una serie de 
fotografías. Este archivo es especial por la diversidad de soportes. Generando un desafío grande para la conservación, para la guarda, porque obviamente cada material requiere de una conservación distinta y una guarda distinta. En el ordenamiento general se realizó una clasificación con cierta lógica, con un sentido en la vida de Adelina. Si bien se discutió mucho en el equipo internamente, y a lo largo del tiempo seguís diciendo esto por ahí podría haberse puesto de otra manera... creo que también hay que ponerse límites y tenemos que tratar de llegar a una clasificación que permita la accesibilidad.

Desde que Adelina donó el archivo siempre estuvo abierto al público, con las restricciones que te mencionaba antes sobre ciertos materiales, por ser documentación sensible. El único tema que tiene la accesibilidad al archivo de Adelina es que como todavía no está terminada su herramienta descriptiva, ni la guarda total de su archivo, tratamos de ofrecer el material al investigador cuando ya sabemos que está limpio y que puede tener una mínima guarda de conservación. Entonces, algunas búsquedas las tenemos que hacer previamente nosotros y sobre eso tratar de orientar e ir viendo qué podemos ofrecer, porque todavía no tenemos la posibilidad de una búsqueda del tipo: "bueno sentate en la computadora y mirá todo el archivo y elegí qué". Eso es parte de lo que venimos trabajando; un trabajo de mucho tiempo. La guarda lleva horas de cortar hojas de papel libre de ácido, de ver qué soporte se le tiene que hacer a cada cosa y qué carpeta y en qué caja va a ir y después completar el inventario puntual de esa carpeta, para después recién pasar a lo otro. Todo eso lleva mucho tiempo, pero es algo que venimos sosteniendo, se va avanzando todos los días. 
María Emilia Nieto. Florencia Lloret y una mirada al fondo documental de Adelina Dematti de Alaye

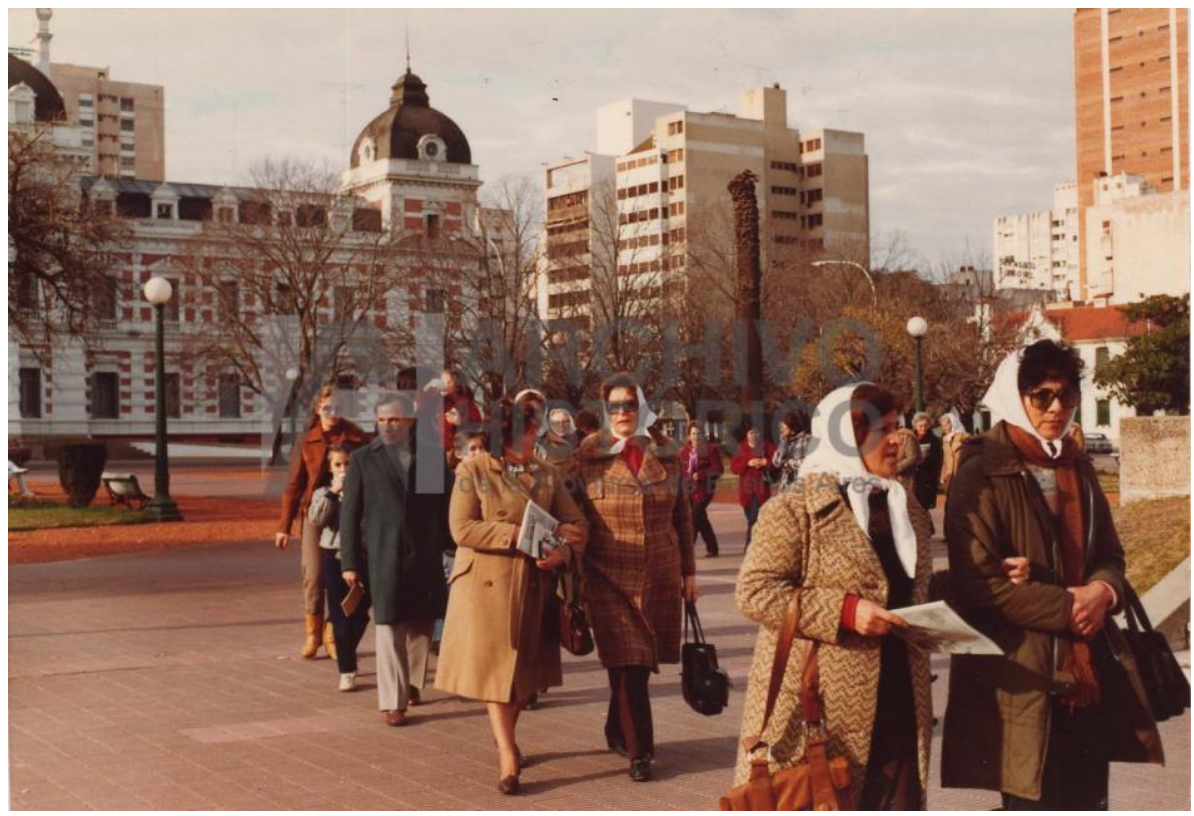

Foto 3. Entrega del petitorio al gobierno provincial, Plaza San Martín, La Plata (Fondo documental de Adelina Dematti de Alaye, integrante de Madres de Plaza de Mayo-La Plata). 1 de julio de 1981.

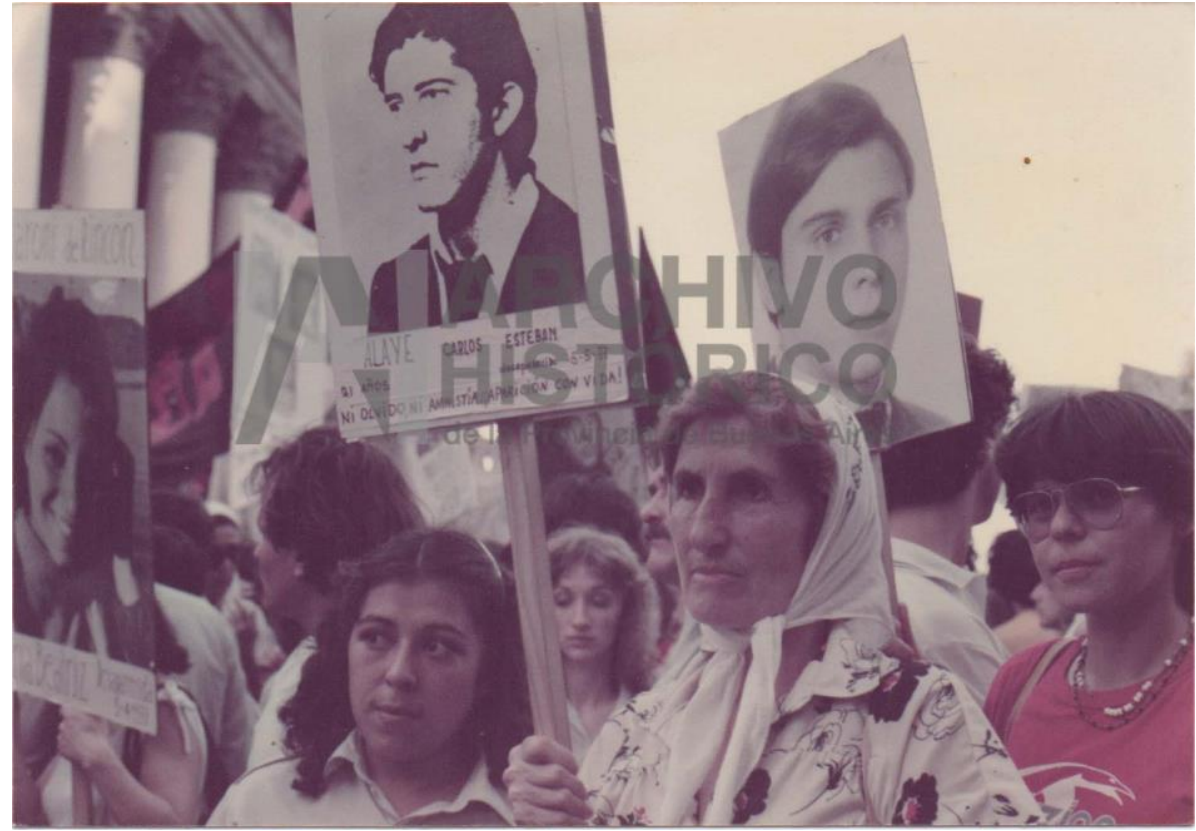

Foto 4. Ronda último jueves de la dictadura. "Frente a la Casa Rosada María Inés y Gladys Riquelme. Fotos: PoseAlaye" (Fondo documental de Adelina Dematti de Alaye, integrante de Madres de Plaza de Mayo-La Plata). 8 de diciembre de 1983. 


\section{— ¿Y quiénes consultan el archivo? ¿Hay consultas de otras instituciones?}

- Han venido por ejemplo de Canal Encuentro, a buscar fotos, porque iban a hacer un documental. Pero en general son investigadores particulares, que están trabajando sobre el tema de Madres ya sea desde la facultad o la universidad. Hay un grupo de fotógrafos que la conocieron a Adelina y vinieron de parte de ella, vienen haciendo un trabajo de investigación y han producido algunos documentales o audiovisuales. El Archivo tiene visitas para colegios o instituciones que lo pidan, visitas guiadas al Archivo Histórico. Estamos incorporando, sobre todo en los colegios secundarios, mostrarles también parte del archivo de Adelina.

Lo que permite el archivo de Adelina son muchos ingresos. Podés no venir a tocar el tema de Madres de Plaza de Mayo o no venir a buscar puntualmente una información sobre Adelina, pero tiene un montón de líneas transversales sobre las cuales podés encontrar cosas. Necesitamos generar una herramienta más elaborada y más extensa y que se pueda digitalizar el material. Lo que es fotografía tenemos todo digitalizado ya, y se están digitalizando negativos, por eso digo que hay un avance continuo, diario, constante, pero que por ahí se pierde en el cotidiano.

Hasta que vivió Adelina fue un archivo abierto, es decir que hasta muy poco antes de morir seguía incorporando documentación. La llamabas para saber cómo estaba y te decía "Pasá a buscar que tengo en mi casa..." Entonces pasabas y tenía dos cajas de papeles que había encontrado, entre las cuales había cosas muy importantes. Hasta que Adelina falleció siempre se lo consideró un archivo abierto, que sufría modificaciones de todo tipo, constantemente. Incluso porque Adelina venía y te pedía cosas, iy si te agarraba desprevenida se llevaba el original! Tenías que andar hasta en una lucha de decir, "Bueno Ade, te saco una copia, o ya está inventariado eso", iy renegaba de eso! Indudablemente ya no va a entrar más documentación por parte de Adelina, sí lo que se empieza a generar son producciones que surgen desde y sobre su archivo y venimos tratando de ver cómo lo vamos a incorporar también.

Hoy en día somos cinco personas que estamos trabajando con el archivo y que tenemos presente la cuestión de dónde están los documentos. Pero lo que nos preocupa sobremanera es que esto tiene que quedar ordenado para que cualquiera pueda tener acceso a la búsqueda. Así que, sobre eso se avanza en trabajo sostenido. 


\section{— ¿Cuál es el aporte que hace el archivo de Adelina a la historia y a la memoria del pasado reciente?}

-Yo siempre parto de la frase que está en el panel, que la dijo Adelina cuando le preguntamos por qué había generado el archivo. "En un primer momento era para mostrárselo a Carlos cuando volviera; luego, para poder contar la historia familiar a mis nietos. Hoy, pienso que siempre fue para el futuro". Y esa guarda, siempre le marcamos a Adelina, no fue a partir de la desaparición de Carlos; ella hizo una guarda anterior, muy intuitiva, de coleccionista.

Creo que el aporte a la época de la dictadura es muy grande, porque la mirada de Adelina era muy particular. Era la mirada de una persona que, si bien a partir de la desaparición de Carlos se convirtió en una persona militante, ella había militado antes otras causas. Cuando ves y escuchas la documentación, encontrás que hubo militancias anteriores en esto de preocuparse por otros, de estar con otros, de buscar con otros. Entonces todo su archivo, si bien tiene un gran desarrollo en lo que está relacionado con la época dictatorial y la vuelta a la democracia y las búsquedas judiciales, tanto en nuestro país como en el extranjero, también tiene una gran posibilidad de entrar por otras líneas. Por ejemplo, la parte de docencia se podría trabajar sacando a la figura de Adelina y hacer una investigación sobre docencia a partir de esa documentación. Hay una cantidad de diarios que guardó y uno podría tomarlos más allá de por qué Adelina guardó esos diarios, hay líneas transversales que permite su archivo. Y un aporte a la memoria, sin duda, a la construcción de memorias. Para nosotros es significativo tener este fondo en el Archivo Histórico, porque nos permite seguir reforzando el sector de Historia Reciente, nos ha permitido trabajar netamente con las memorias de Adelina, ya no sólo el documento sino con la producción de entrevistas de historia oral; es haber trabajado con sus memorias, con cómo ella construyó sus memorias a lo largo del tiempo, y toda esta tarea que nos ha permitido hacer; de relacionar los documentos, los papeles que guardó, con el sentido que ella les daba. Creo que ese aporte es magnífico. Sigo leyendo cosas y sigo ordenando, y mirando papeles y descubriendo cosas, sigo fascinándome y digo cómo se le ocurrió guardar esto y cómo se arriesgó, en otros casos a pedir una copia de, o a guardar. Creo que hay mucho trabajo para los investigadores, y cuando digo investigadores, digo en general, quien quiera venir a consultarlo creo que tiene posibilidad de lecturas diversas. Es un aporte inmenso. 


\section{Notas}

(1) Entrevista llevada a cabo el 12 de julio de 2017. Agradezco a Florencia Lloret por su compromiso con la realización de la misma.

(2) Campo de la Historia que aborda el pasado reciente -período que la historiografía positivista no considera como objeto de investigación- asociado a la violencia política, regímenes dictatoriales y represión, y donde la metodología de Historia oral tiene un lugar privilegiado.

(3) Carlos Esteban Alaye, hijo de Adelina Dematti de Alaye, fue secuestrado el 5 de mayo de 1977. Era estudiante de psicología, obrero metalúrgico, delegado gremial y militante de Montoneros. 